\title{
Comunicación
}

\section{PRESENCIA DE Hepatozoon caimani (APICOMPLEXA: HEPATOZOIDAE) EN EL “CAIMÁN BLANCO” Caiman crocodilus (LINNEUS 1758) EN UN ZOOLÓGICO DE LIMA, PERÚ}

\author{
Presence of Hepatozoon caimani (Apicomplexa: Hepatozoidae) in the \\ "Spectacled Caiman" Caiman crocodilus (Linneus 1758) in a Zoo of \\ Lima, P ERU
}

\author{
Gianmarco Rojas $^{1,2}$, Rafael Alvis ${ }^{3,4}$, José Pino $^{3}$, Betty Shiga ${ }^{3}$
}

\section{Resumen}

Se evaluó la posible ocurrencia de Hepatozoon caimani en una población de "caimán blanco" Caiman crocodilus en un zoológico de Lima, Perú. Se colectó muestras de sangre periférica de 10 especímenes de ambos sexos y de diversas edades, clínicamente sanos y mantenidos en cautiverio en el Parque Zoológico Huachipa. Se obtuvo tres frotices de sangre por animal y fueron teñidos con May-Grunwald-Giemsa 2\%. Se identificó la presencia de hemoparásitos intraeritrocitarios del género Hepatozoon, determinándose por sus aspectos morfológicos que los gamontes corresponden a H. caimani.

Palabras clave: Alligatoridae, Caiman crocodilus, Hepatozoon caimani

\section{Abstract}

The presence of Hepatozoon caimani in a population of Caiman crocodilus in a zoo located in Lima, Peru was evaluated. Blood samples were collected from 10 specimens of both sexes and various ages. The animals were clinically healthy and kept in captivity at the "Parque Zoológico de Huachipa". Three blood smears were prepared per animal and stained with May-Grunwald-Giemsa 2\%. Intraerythrocitic haemoparasites of the genus Hepatozoon were identified and due to the morphological aspect they were considered to be H. caimani.

Key words: Alligatoridae, Caiman crocodilus, Hepatozoon caimani

\footnotetext{
${ }^{1}$ Parque Zoológico Huachipa, Lima. E-mail: gianmarco_rojas@yahoo.com

${ }^{2}$ Facultad de Medicina Veterinaria y Zootecnia, Universidad Científica del Sur, Lima

${ }^{3}$ Laboratorio de Reproducción y Biología de Desarrollo, Facultad de Ciencias Biológicas, Universidad Nacional Mayor de San Marcos, Lima

${ }^{4}$ Laboratorio de Biología, Universidad Científica del Sur, Lima
} 
Las hemogregarinas son esporozoarios, parásitos intracelulares, que se encuentran en vertebrados de sangre fría, y pueden producir hipertrofia de los glóbulos rojos del huésped parasitado (Ball et al., 1969; Beyer, 1977). Una infección parasitaria intensa en el Caiman crocodilus yacare (Yacaré del Pantanal), puede causar serios daños, especialmente por la obstrucción de los capilares, como consecuencia de una trombosis y necrosis tisular (Souza Lima y Silva, 2004). Usualmente, se observa uno o dos parásitos de forma ovalada y con extremos curvados dentro de un eritrocito, el cual puede estar distorsionado en forma y tamaño.

Chatton y Roubaud (1913) describieron en África la esporogonia de una especie de Hepatozoon, encontrada en la mosca tse-tsé, Glossina palpalis, y sospecharon que el parásito se había alimentado de un lagarto o un cocodrilo. Hoare (1932) encontró ooquistes multisporocísticos de hemogregarinas en la sangre de G. palpalis que se habían alimentado con la sangre de cocodrilo del Nilo Crocodylus niloticus. Durante años, los hemogregarinas reportados en la sangre de reptiles, aves y mamíferos fueron asignados al género Haemogregarina, como resultado de la similitud morfológica de los gamontes de hemogregarinas de diversas especies (Smith, 1996, citado por Moço, 2008). En los últimos años se ha sugerido que todos los hemogregarinas encontrados en los cocodrilos deben ser transferidos al género Hepatozoon (Siddall, 1995: Smith, 1996); sin embargo, H. pettiti y H. caimani fueron considerados como una única especie. Parece ser que hay suficientes evidencias que demostrarían que estos son estadios esporogónicos, característicos de este género (Hoare, 1932; Pessôa et al., 1972). H. caimani ya fue descrito en Caiman yacare, C. latirostris y $C$. crocodilus en Brasil.

Las hemogregarinas son transmitidas a través de un vector invertebrado, como las sanguijuelas y los artrópodos; siendo los cocodrilianos los hospederos definitivos. Más de 300 especies han sido encontradas en cocodrilos clínicamente normales, incluyendo el Haemogregarina crocodilinorum en aligatores americanos Aligator mississipiensis (Lloyd, 2003). Por otro lado, en la mayoría de los casos de animales en cautiverio, la transmisión es autolimitante (Jacobson, 1986).

El "Caimán blanco" Caiman crocodilus es la especie de aligatórido más común y de mayor distribución en América Latina. Geográficamente ocupa territorios ribereños tropicales desde el sudoeste de México hacia el noroeste de Argentina (Grzimek, 1975), principalmente en la región amazónica.

En las últimas décadas, debido al incremento del valor económico de los productos obtenidos en las especies de la Familia Crocodilidae, así como por la posibilidad de realizar estudios filogenéticos, se ha despertado gran interés en realizar trabajos de investigación en estos animales (Oliveira et al., 1998). Sin embargo, pocos trabajos científicos describen la morfología de las células sanguíneas de los cocodrilos y aligatóridos (Oliveira et al., 1998; Souza-Lima y Silva, 2004; Ortiz, 2008).

El objetivo del presente estudio fue evaluar la posible ocurrencia de $H$. caimani en una población de "caimán blanco" $C$. crocodilus en el Parque Zoológico Huachipa, ubicado en el distrito de Ate-Vitarte, provincia y departamento de Lima, Perú.

En el verano de 2006, se muestrearon 10 individuos de $C$. crocodilus, de ambos sexos (5 machos y 5 hembras) y de varias edades (4 juveniles y 6 adultos), clínicamente sanos, provenientes de la región Amazónica del Perú y mantenidos en cautiverio en las instalaciones del Parque Zoológico desde el año 2000. El alojamiento de estos animales está conformado por un área seca, con substrato de tierra y un estanque con agua potable corriente, sin techo. Los animales están expuestos a dípteros hematófagos y a sanguijuelas durante algunas estaciones del año; 

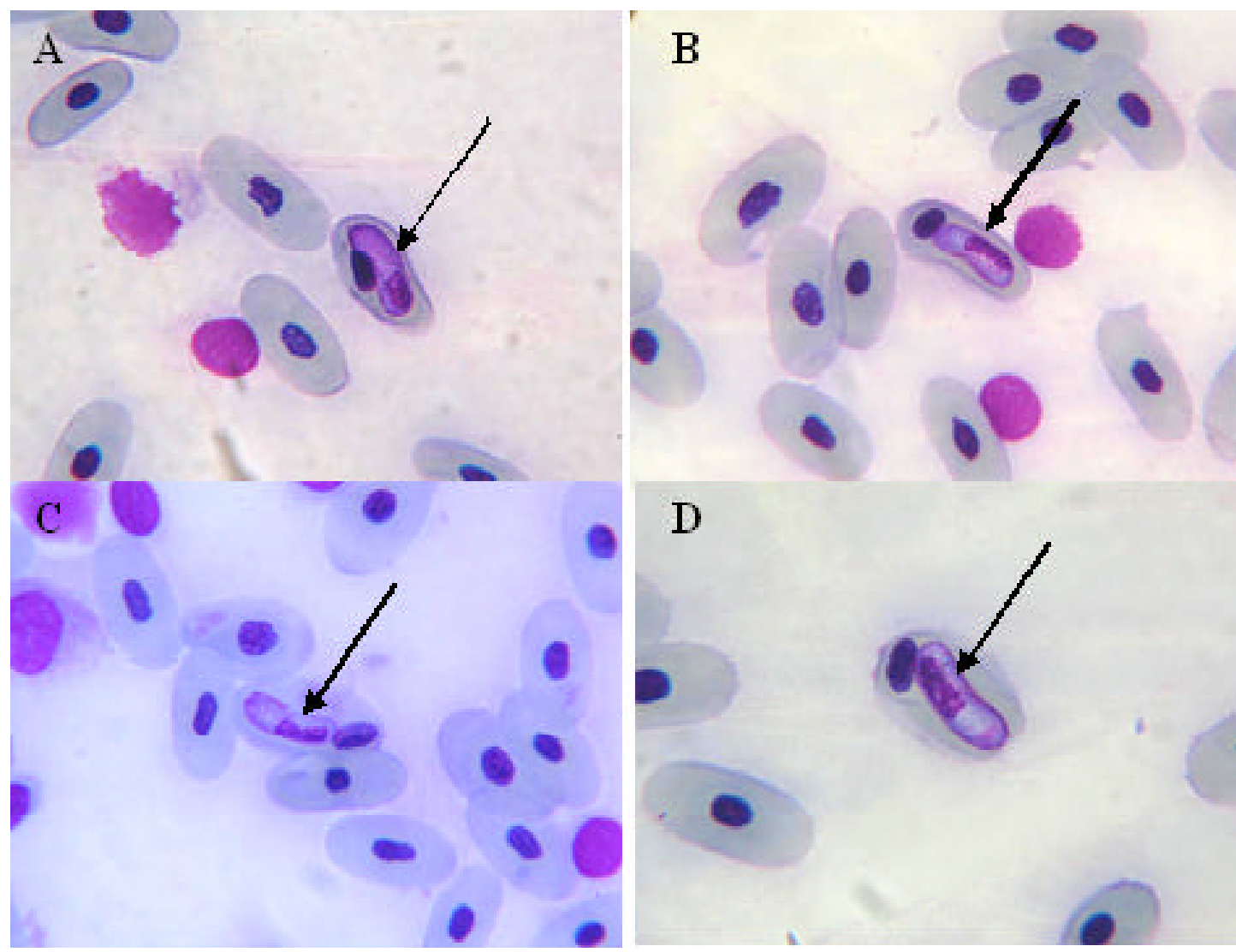

Figura 1. Hepatozoon caimani (flecha) parasitando eritrocitos de C. crocodilus (Coloración May-Grunwald-Giemsa): A, B, C: 630X; D: 1000X.

sin embargo, no se ha confirmado su actividad como vectores de $H$. caimani.

Los animales fueron contenidos físicamente por sujeción manual con ayuda de cuerdas. Se pesaron y se les aplicó vitaminas y antiparasitarios. Se les colectó una muestra de sangre por punción venosa en el seno postoccipital, ubicado entre el final del cráneo y la placa más gruesa de la nuca (Campbell, 1996). Todos los muestreos se realizaron en el mismo día.

Se prepararon tres frotices de sangre por cada individuo. Los frotices se dejaron secar al aire, se fijaron en metanol absoluto por 10 minutos y fueron teñidos con el colorante MayGrunwald-Giemsa $2 \%$ por 15 minutos. Las láminas se observaron al microscopio óptico de campo claro en el Laboratorio de Reproducción y Biología de Desarrollo de la Facultad de Ciencias Biológicas, Universidad Nacional Mayor de San Marcos, Lima. Todos los hemoparásitos identificados como $H$. caimani se midieron con ayuda de un ocular micrométrico.

Se encontró un individuo (10\%) positivo a la presencia de hemoparásitos, llegándose a contar 12 parásitos intraeritrocitarios en el frotis (Fig. 1). El animal infestado fue una hembra adulta, identificada con microchip N. ${ }^{\circ} 075 * 275 * 261$, de buena condición corporal y sin alteraciones clínicas aparentes; sin embargo, en el análisis hematológico presentó un nivel bajo de hematocrito $(6.4 \mathrm{~g} / \mathrm{dl})$ $\mathrm{y}$ hemoglobina (22\%). 
En el frotis se observó eritrocitos conteniendo gamontes de H. caimani (Fig. 1). Los hemoparásitos no mostraban dimorfismo sexual y solo se encontraron dentro de los glóbulos rojos maduros, cuyo núcleo quedó polarizado por la presencia del gamonte. Alrededor del parásito se evidenció una envoltura o membrana basófila con varios grados de intensidad de color. La forma típica del parásito es alargada y de extremos redondeados, observándose en forma paralela al eje longitudinal del eritrocito. El citoplasma es ligeramente basófilo y heterogéneo, y el núcleo tiende a ser lateral o acéntrico, se tiñe fuertemente y tiene una apariencia granular y densa

La longitud promedio para los gamontes H. caimani fue de $13.25 \mathrm{~m} \mu$ y el ancho de $4.25 \mathrm{~m} \mu \mathrm{Q}$. Medidas cercanas a otros reportes de $H$. caimani en el caimán blanco (12.15 x $4.3 \mathrm{~m} \mu$, Lainson et al., 2003) y Caiman yacare $(12.70 \times 4.4 \mathrm{m \mu}$, Viana y Marques, 2005).

Los criterios taxonómicos para la identificación de Hepatozoon sp se basan principalmente en la caracterización morfológica de los gamontes en la sangre del huésped vertebrado y de los estadios esporogónicos del huésped invertebrado. El hemoparásito en este estudio fue identificado como $H$. caimani, (Souza-Lima y Silva, 2004); asimismo, el estadio encontrado correspondió a un gametocito (Souza-Lima y Silva, 2004). Si bien se ha establecido mundialmente que todos los hemogregarinos encontrados en crocodilianos pertenecen al género Hepatozoon, se requiere ampliar los estudios del ciclo esporogónico en el huésped invertebrado para una mejor clasificación (Lainson et al., 2003).

Se sabe que, en condiciones normales, el Hepatozoon sp. convive con sus hospederos reptiles sin causar trastornos clínicos ni patológicos evidentes, lo cual explica que los valores de hematocrito y hemoglobina del caimán infectado, si bien ligeramente dismi- nuidos, estaban dentro de los valores encontrados en el resto de la población muestreada (hematocrito: $8.2 \pm 1.5 \%$; hemoglobina: 27.4 $\pm 3.9 \mathrm{~g} / \mathrm{dl}$ ). Sin embargo, es importante mencionar que en serpientes bajo condiciones de estrés e inmunosupresión, se han reportado cuadros neurológicos y de anemia hemolítica, y que en los hallazgos de necropsia se pueden asociar a la presencia de pancreatitis, esplenitis y hepatitis necrosante (Moço, 2008).

La presencia de $H$. caimani en un individuo de $C$. crocodilus, mantenido en cautiverio por más de 6 años, confirma que en condiciones adecuadas de manejo y de bajo estrés, como son las condiciones de cautiverio dentro de las instalaciones del Parque Zoológico Huachipa, el Hepatozoon puede convivir sin causar mayores problemas a su hospedero.

\section{Agradecimientos}

Se agradece al personal del área de fauna del Parque Zoológico Huachipa, destacando el apoyo de Lizette Bermúdez y del equipo de manejo de reptiles. A Germán Chávez y Sibylle Durán por su interés en el estudio, y al personal del Laboratorio de Reproducción y Biología de Desarrollo de la Facultad de Ciencias Biológicas, UNMSM, por su apoyo en la revisión de las muestras de laboratorio.

\section{Literatura Citada}

1. Ball G, Chao J, Telford Jr S. 1969. Hepatozoon fusifex sp. n., a hemogregarine from Boa constrictor producing marked morphological changes in infected erythrocytes. J Parasitol 55: 800-813.

2. Beyer T. 1977. Electron microscope study of Karyolysus sp. (Sporozoa: Adeleida: Haemogregarinidae) and of changes induced in the infected host cell. Protistologia 13: 57-66. 
3. Campbell TW. 1996. Clinical pathology. In: Mader DR (ed). Reptile medicine and surgery. Philadelphia: WB Saunders. $p$ 474-483.

4. Chatton E, Roubaud E. 1913. Sporogonie d'une hémogrégarine chez une tsétsé (Glossina palpalis R. Desv.). Bull Soc Pathol Exot 6: 226-233.

5. Grzimek B. 1975. The crocodiles and alligators. In: Grzimek's animal life enciclopedia. New York: Van Noytrand Reinhold. p 124-146.

6. Hoare C. 1932. On protozoan blood parasites collected in Uganda, with an account of the life cycle of the crocodile haemogregrine. Parasitology 24: 210-224.

7. Jacobson E. 1986. Parasitic diseases of reptiles. In: Fowler ME. (ed). Zoo \& wild animal medicine. $2^{\text {th }}$ ed. Philadelphia: WB. Saunders. p 162-181.

8. Lainson R, Paperna I, Naiff R. 2003. Development of Hepatozoon caimani (Carini, 1909) Pessôa, De Biasi \& De Souza, 1972 in the caiman Caiman c. crocodylus, the frog Rana catesbiena and the mosquito Culex fatigans. Mem Inst Oswaldo Cruz 98: 103-113.

9. Lloyd M. 2003. Crocodilia (Crocodiles, alligators, gavials, and caimans). In: Fowler ME, Miller RE (eds). Zoo \& wild animal medicine. $5^{\text {th }}$ ed. Philadelphia: WB Saunders. p 59-70.

10. Moço T. 2008. Prevalência de Hepatozoon spp em serpentes e caracterização morfológica, morfométrica e molecular de Hepatozoon spp
(Apicomplexa, Hepatozoidae) de Crotalus durissus terrificus (Serpentes, Viperidae) naturalmente infectadas. Tesis de Mestre. UNESP. 77 p.

11. Oliveira L, Moura W, Matushima E, Egami M. 1998. Características citoquímicas morfológicas y ultrastructurales de eosinófilos de Caiman crocodilus yacare (Daudin, 1802) (Reptilia, Crocodilia). Rev Chil Anat 16: 245-254.

12. Ortiz L. 2008. Presencia de Hepatozoon sp. en caimán blanco Caiman crocodilus (Linneaus 1758) mantenidos en cautiverio en el Parque Zoológico Quistococha (Loreto, Perú). Tesis de Bachiller. Facultad de Veterinaria y Zootecnia. Universidad Peruana Cayetano Heredia. 17 p.

13. Siddall M. 1995. Phylogeny of adeleid blood parasites with a partial systematic revision of the haemogregarine complex. J Euk Microbiol 42: 116-125.

14. Smith T. 1996. The genus Hepatozoon (Apicomplexa: Adeleina). J Parasitol 82: 565-585.

15. Souza-Lima ES, Silva RA. 2004. Aspectos morfológicos do parasita Hepatozoon caimani em Jacaré (Caiman crocodilus yacare) no Pantanal Sul. Comunicado Técnico 41. Corumbá, Brasil: Embrapa. 4 p.

16. Viana L, Marques E. 2005. Haemogregarine parasites (Apicomplexa: Hepatozoidae) in Caiman crocodilus yacare (Crocodilia: Alligatoridae) from Pantanal, Corumbá, MS, Brazil. Rev Bras Parasitol Vet 14(4): 173-175. 